The trial investigators have also begun a detailed cost-effectiveness analysis, to aid in preparing guidelines for the use of EVAR in routine clinical practice.

Claire Braybrook

Original article EVAR trial participants (2005)

Endovascular aneurysm repair versus open repair in patients with abdominal aortic aneurysm (EVAR trial 1): randomised controlled trial. Lancet 365:2179-2186

\section{Management of abdominal aortic aneurysm in patients unfit for open repair surgery: EVAR trial 2}

The endovascular aneurysm repair (EVAR) technique was developed to help manage patients with large abdominal aortic aneurysms who were considered unfit for open repair surgery. The EVAR trial 2 assessed patient outcomes following EVAR surgery in terms of all-cause mortality, aneurysm-related mortality, health-related quality of life, postoperative complications and hospital costs.

Patients aged 60 years or older with an aneurysm of at least $5.5 \mathrm{~cm}$ in diameter were referred from any of 31 UK hospitals, with 166 patients randomized to undergo EVAR and 172 patients to receive no surgical intervention.

In the EVAR group, the actual 30-day operative mortality was $7 \%$, while the intention-totreat mortality was $9 \%$ because of aneurysm ruptures before surgery. Over 4 years, there were no statistically significant differences in all-cause mortality or aneurysm-related mortality between the EVAR and no-intervention groups. Reintervention was necessary in $26 \%$ of patients in the EVAR group compared with $4 \%$ in the no-intervention group. Mean hospital costs were also higher for patients who underwent EVAR surgery compared to those who did not. No differences in health-related quality-of-life scores were reported between the two groups.

In conclusion, EVAR does not improve survival in patients deemed unfit for open repair surgery, and is associated with an increased risk of postoperative complications and high hospital costs.

Claire Braybrook

Original article EVAR trial participants (2005)

Endovascular aneurysm repair and outcome in patients unfit for open repair of abdominal aortic aneurysm (EVAR trial 2): randomised controlled trial. Lancet 365: 2187-2192

\section{After ischemic stroke, patients are at higher risk of recurrent stroke than of cardiac events}

A recent retrospective study of patients who had suffered a first ischemic stroke or transient ischemic attack (TIA), investigated the relative incidence of subsequent cerebrovascular and cardiac events.

Brown et al. investigated the hospital records of 1,923 male and female patients (mean age 77) who had suffered an ischemic stroke or TIA, and recorded the nature of the first cardiac or cerebrovascular adverse event to occur during a 30-month follow-up period. Adverse events noted were cardiac events, myocardial infarction, percutaneous transluminal coronary angioplasty, coronary artery bypass grafting, and ischemic stroke. Cumulative incidence estimates compared cardiac events and stroke as secondary events.

During the follow-up period, 239 patients had a stroke as their first event, and 172 patients had a cardiac event (10 patients suffered both a stroke and cardiac event on the same day). Recurrent strokes were more frequent than cardiac events at all times during the followup period-at 30 days, $2.5 \%$ and $0.8 \%$ of patients had suffered a stroke and cardiac event, respectively. By 6 months this had risen to $6.6 \%$ and $2.6 \%$, and at 2 years $11.8 \%$ had suffered a stroke, and $7.7 \%$ a cardiac event.

The authors conclude that the risk of having a stroke following an initial stroke or TIA, is greater than the risk of having a cardiac event, and that consequently there are implications for the selection of appropriate prophylactic therapy.

Christine Kyme

Original article Brown DL et al. (2005) Recurrent stroke risk is higher than cardiac event risk after initial stroke/transient ischemic attack. Stroke 36: 1285-1287

\section{Does coating stents with titanium oxynitride reduce restenosis?}

Restenosis due to neointimal proliferation is a major limitation of percutaneous coronary interventions. Stent surface material can influence restenosis rates, with gold-coated stents leading to an increased risk of restenosis; similarly, stainless steel stents increase the 